\title{
CO Kinematics, Star Formation and Dynamical Evolution of the Lensed Starburst NGC 4102
}

\author{
Shardha Jogee and Jeffrey D. P. Kenney \\ Yale University, Astronomy Department, New Haven, CT 06520-8101
}

\section{Introduction}

The circumnuclear (inner kiloparsec) regions of spiral galaxies are time-dependent systems whose morphology and dynamics can change significantly over less than a Hubble time. To develop an insight into this evolution it is important to study the fate of gas driven towards the central parts of a galaxy especially near the dynamical resonances. The panoply of $\mathrm{CO}$ morphologies displayed by the circumnuclear regions of starburst and nonstarburst galaxies such as NGC 3504, NGC 3351, NGC 7479, NGC 6951, etc, (Kenney, these proceedings) is not yet clearly understood. Another unsettled issue concerns the criteria for star formation in the circumnuclear region where, in contrast to the outer disk, the rotation curve is nearly solid-body and rises rapidly. These issues need to be resolved; the gas mass fraction, the radial variation of SFR (star formation rate) and its interplay with the gas kinematics can shed light not only on the temporal evolution of the circumnuclear $\mathrm{CO}$ morphology but also on secular evolution through the destruction and creation of new stellar components. For instance, stellar bars can be destroyed by an increase in the central gas mass concentration of a few \% of the total galaxy mass while mechanisms to build or enhance a bulge by vertical scattering of the stars in a compact circumnuclear stellar disk have been proposed (Sellwood 1994, Pfenniger \& Norman 1990). We have carried out a study of the circumnuclear region of NGC 4102 with these important issues in mind. NGC 4102, a LINER/HII, SABb spiral galaxy at a distance of $17 \mathrm{Mpc}$ with a bar and/or lens feature qualifies as one of the most luminous nearby starbursts (Devereux 1989) and seems to be in a very early phase of evolution. The outflow timescale of the central starburst wind in NGC 4102 , determined from optical spectroscopy of the ionized swept-up gas, is $10^{6}$ years (Boer 1994), suggesting it is at an earlier evolutionary phase than M82 and NGC 253 whose outflow timescales are significantly larger.

\section{Observations and Discussion}

\subsection{The Intrinsic Distribution, Velocity Field and Velocity Disper- sion of the Molecular Gas}

The circumnuclear region of NGC 4102 was observed in the $\mathrm{CO}(\mathrm{J}=1 \rightarrow 0)$ line with the OVRO millimeter-wave interferometer at a resolution of $2^{\prime \prime}$. After data calibration, uniformly-weighted moment maps were produced with the NRAO AIPS software. We estimated the true velocity field, velocity dispersion, and surface brightness by correcting the moment maps for beam smearing effects 

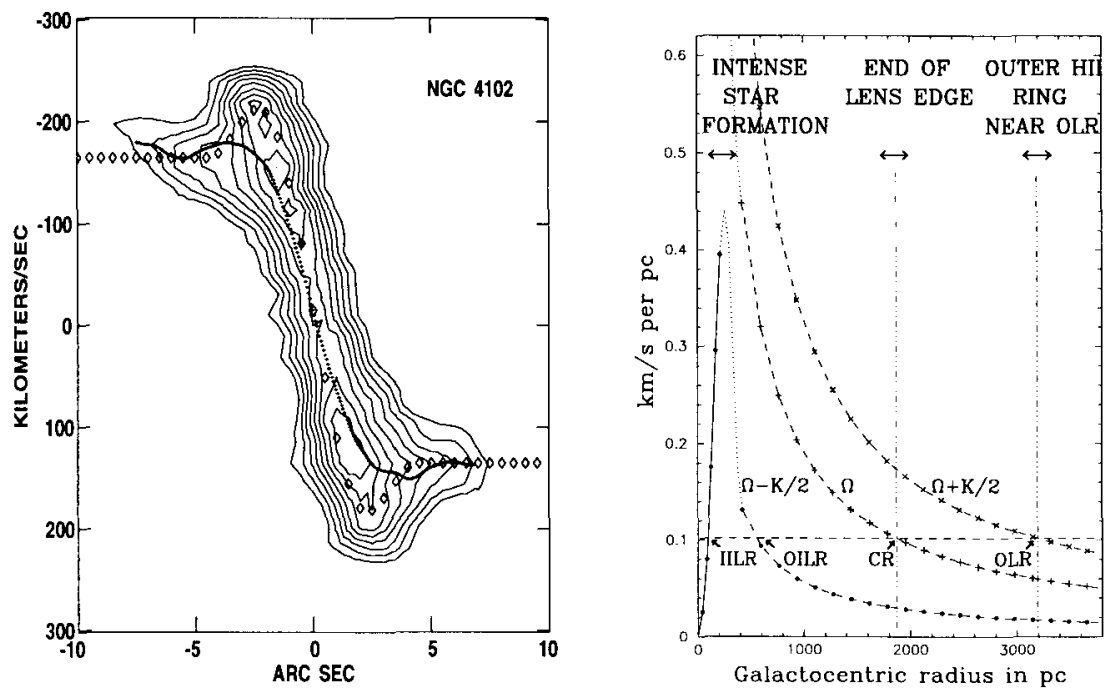

Figure $1 . \quad$ The model rotation curve $(\diamond)$ and moment 1 slice $(\cdot)$ superposed on the CO spatial velocity plot of NGC 4102 along the kinematic major axis (left).

Figure 2. The relation between the dynamical resonances, the starburst activity, the lens feature and the outer HII ring (right).

using an iterative modelling procedure (Jogee \& Kenney 1995). The position angle and inclination of the compact $\mathrm{CO}$ disk coincide with that of the outer large-scale disk. The distribution of molecular gas shows significant deviations from axisymmetry, suggesting a modest, loosely-wound spiral-like morphology in the central $200 \mathrm{pc}$. However, the velocity field in the central $200 \mathrm{pc}$ is consistent with purely circular motion. At larger radii, around $3^{\prime \prime}$, the spatial velocity plot (Figure 1) shows evidence of a sharp discontinuity in the kinematics, which could be due to streaming motion along the bar (or lens).

\subsection{Dynamical Resonances and Global Morphology of NGC 4102}

$\mathrm{H} \alpha$ and $R$-band observations were made with the KPNO $0.9 \mathrm{~m}$ telescope. The $R$-band image shows a steeply declining surface brightness profile in the central $500 \mathrm{pc}$ followed by a region extending over 600 to $1900 \mathrm{pc}$ where the surface brightness first declines slowly then falls sharply. The light profile of this region is consistent with the plateau and sharp edge of a lens. In order to determine the location of the resonances, we use a rotation curve whose shape in the circumnuclear region is derived from the $\mathrm{CO}$ data while in the outer regions, it is assumed to be flat with a value consistent with the observed HI linewidths. Assuming the Corotation Resonance is near the outer edge of the lens feature, the location of the Outer Lindblad Resonance (OLR) coincides with the outer HII ring (Figure 2). The dust lanes in the inner kiloparsec of NGC 4102 are 
offset from the nucleus, confirming the presence of Inner Lindblad Resonances (ILRs). The location of the Outer ILR (OILR) obtained from the dust lane morphology is consistent with the kinematic estimate.

\subsection{Star Formation Activity and Dynamical Evolution of the Star- burst}

The radio continuum, $\mathrm{H} \alpha$ and $\mathrm{CO}$ data reveal that the SFR per unit gas mass is high in the central $150 \mathrm{pc}$ but significantly drops where there is a discontinuity in the gas kinematics. The region of strong star formation is definitely inside the OILR and may extend inside the Inner ILR (IILR). In the region of star formation, the Toomre Q parameter is close to 1 . In many respects, NGC 4102 is similar to the young starburst NGC 3504 (Kenney et al. 1993).

The short outflow timescale and compact $\mathrm{CO}$ morphology suggest NGC 4102 is a young starburst. The CO morphology will evolve from centrally peaked to ring-like as star formation consumes and starburst winds expel the gas more quickly in the central few hundred pc. The compact $\mathrm{CO}$ disk will turn into a compact circumnuclear stellar disk harboring a significant young population. The lens feature of NGC 4102 might be related to the large contribution (at least $40 \%$ ) of the gas mass to the dynamical mass in the inner $500 \mathrm{pc}$. It has been suggested that lenses, which are poorly understood components, could be the remnant of bars destroyed by an increase in the central mass concentration instilled by gas driven to the center of a galaxy (Combes, Freeman, this conference). NGC 4102 might well be currently exemplifying such a process.

\section{Conclusions}

Intense star formation in NGC 4102 is occuring inside the OILR, in a region where the motions of the molecular gas are predominantly circular. Beyond this region, the kinematics show evidence of a sharp discontinuity and the SFR per unit gas mass drops significantly. The Toomre $Q$ parameter is close to 1 in the region of star formation; this suggests that the simple gravitational instability picture which has been successfully used to explain cutoffs in star formation in the outer disks of spiral galaxies could be applicable to the inner circumnuclear CO disk. The circumnuclear region of NGC 4102 harbors a high gas mass fraction and seems to be undergoing significant morphological evolution.

\section{References}

Boer, B. 1994, in Mass Transfer Induced Activity in Galaxies, I. Shlosman, Cambridge: Cambridge Univ. Press, 308

Devereux, N. A. 1989, ApJ, 346, 126

Jogee, S. \& Kenney, J. D. P. 1995, in preparation

Kenney, J. D. P., Carlstrom, J., \& Young, J. S. 1993, ApJ, 418, 687

Pfenniger, D. \& Norman, C. 1990, ApJ, 363, 391

Sellwood, J. A. 1994, in Numerical Simulations in Astrophysics, J. Franco, Cambridge: Cambridge Univ. Press, 1 